\title{
PERFORMANCE ANALYSIS OF VRLA BATTERIES UNDER CONTINUOUS OPERATION
}

\author{
J.Vishnupriyan $^{1}$, P.S.Manoharan ${ }^{2}$, V.Seetharaman ${ }^{3}$ \\ ${ }^{I}$ P.G. Student, Electrical and Electronics Engineering, Thiagarajar College of Engineering, Tamilnadu, India \\ ${ }^{2}$ Associate Professor, Electrical and Electronics Engineering, Thiagarajar College of Engineering, Tamilnadu, India \\ ${ }^{3}$ Assistant Professor, Electrical and Electronics Engineering, Thiagarajar College of Engineering, Tamilnadu, India
}

\begin{abstract}
This paper contributes about the testing and performance improvement analysis of charging, discharging and lifespan characteristics of various capacities rating valve regulated lead acid (VRLA) battery banks under continuous operation. Measurement of basic parameters such as each cell voltages in battery bank, terminal voltage, load current and actual working temperature of battery banks are described in this paper. By analyzing electrical and thermal characteristics of various capacity rating battery banks, the best battery bank can be offered for particular emergency run time applications. The experimental result analysis of $48 V$, VRLA batteries in potential application performances at temperature sensitivity in the range of $+22^{\circ} \mathrm{C}$ to $+52^{\circ} \mathrm{C}$ have been determined. This study presents data on $80 \%$ depth of discharge (DOD) life test is performed on nominal capacities of 300Ah, 550Ah, 577Ah, 660Ah and 682Ah battery banks for the test duration of 32 to 43 hours. The purpose of this study is to validate the life characteristics of VRLA batteries and develop baseline information on failure rates and mechanisms. The data from this experiment on 80\% DOD is performed on laboratory VRLA batteries and this model is used for real time applications. This information is important in the ongoing development of long life batteries.
\end{abstract}

Keywords: Valve Regulated Lead Acid (VRLA) battery, Battery Bank (BB), Battery reformation, Battery Measurement System (BMS), Depth of Discharge (DOD), Sulfation, Lifespan.

\section{INTRODUCTION}

The main objective of this work is to analyze the performance of charging, discharging and lifespan characteristics of VRLA batteries. Industry consumers are often concerned about backup performances of batteries. But manufacturers give more attention to electrical and thermal characteristics. This is because the battery bank capacity and lifespan of battery is mainly depends on its operating temperature and lead-sulphate crystals deposition on the cell electrodes; thermal characteristics are also to be considered. The presence of bad/death cell in the battery bank, it reduces the ampere-hour rating of the battery bank capacity.

The commercially available battery regenerator is used to reduce the production of sulfation present in the cell electrodes and battery measurement system (BMS) is also used to detection of bad/death cell in the battery bank at experiment time. According to data consumed up to $50 \%$ of total energy consumption to maintain battery temperature at around responsibility is statistics, cooling system has $25^{\circ} \mathrm{C}$ by air conditioner. As an electrochemical product, VRLA battery is sensitive on ambient temperature. The service life of VRLA battery is reduces when the ambient temperature increases $10^{\circ} \mathrm{C}$ [3]. The power battery module under high-rate discharge condition that produces thermal aggregation phenomena. Due to thermal aggregation, generated heat during charging and discharging that distributes in battery module inconsistently affects performance of battery and so that shortens its life [4].

The analysis of unloading and loading characteristics of temperature sensitivity in a range of $-15^{\circ} \mathrm{C}$ to $+50^{\circ} \mathrm{C}$ determined [5]. This charge discharge variation for determining the precision battery parameters such as voltage and current limits and permissible state-of-charge [6]. The analyzed results of YUASA battery laboratories indicated that the positive active material is the major cause for the low discharge performance of the batteries tested. In low performance positive plates tested, in charged condition, $5.52 \%$ of lead sulphate was found and that could be limits the active material available to accept current and so bring down the efficiency of batteries [7].

The charge and discharge experimental curves of Li-Polymer and Ni-MH battery performance has voltage and current versus time at different temperatures are given for temperatures ranging from $-40 \hat{\mathrm{A}}^{\circ} \mathrm{C}$ to $80 \hat{\mathrm{A}}^{\circ} \mathrm{C}$ [8]. The depth of discharge (DOD) affects the cycle life of Lithium-MetalPolymer (LMP) batteries. The main impact of cycling at different DOD"s on LMP battery cycling performances was investigated [9]. $90 \%$ of all alternative energy sources store 
the excess generated energy in battery energy storage systems (BESS) for renewable energy applications [10]. Sulfation is an electrochemical reaction that reduces the electrolyte concentration and as a consequence the cell voltage is also reduced. When a battery is charging and discharging, leadsulphate crystals gradually build up on the cell electrodes that is given in Equation (1), preventing the battery from effectively delivering current to the load. These crystals lead to suffocate the battery. A battery will be unrecoverable if the active material has been lost from the plates or if the plates are bent due to over temperature or over charging [2].

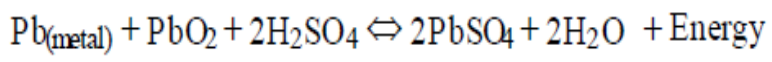

This lead-sulphate increases internal resistance and decrease the weight of electrode. The process of sulfation build up is unavoidable. The performance of the battery varies significantly with temperature [2]. Therefore, precise and rapid measurements are absolutely necessary. The battery regenerator (reformer) is used for desulfate the sulfate crystals present in the cell electrodes. The battery measurement system (BMS) is used to measure voltages of each cells connected in the battery bank in a single measurement. This measurement indicates that how efficiently the each cell produce its output voltage at discharge time. Both total charging and discharging efficiency are major criteria for determining the efficiency of cells in battery banks. As the name BMS indicates, it monitors the total output voltage, each cell voltages and identify the bad cell connected in the battery bank at real time environment. This proposed method of reformer and BMS are used to identify the bad/death cells in a measurement and extending the lifespan of battery by after regeneration of the particular sulfated battery. The measurement is fast and it does not require large space and also very less load fluctuations. The system description of experimental setup is discussed in section 2. The results and discussion of performance analysis of batteries is discussed in section 3 .

\section{SYSTEM DESCRIPTION}

\subsection{Battery Regeneration}

The term reformer represents that battery regeneration and it is commercially available. It is designed to improve the performance of degraded batteries and to extend the battery lifespan. The reformer enables high precise control of AC power, and creates shark pulse to regenerate the degraded secondary lead-acid battery or Ni-Cd battery. Desulfation (Reformation technique) is the process of reversing the sulfation of a lead-acid battery. Desulfation is achieved by high current pulses cycles $(150 \mathrm{~Hz}$ per cycle) produced between the terminals of the battery bank [1]. This technique is also called as pulse conditioning, breaks down the sulphate crystals that are formed on the battery plates.
Short high current pulses tend to work best. Electronic circuits are used to regulate the pulses of different widths and frequency of high current pulses. These can also be used to automate the process since it takes a long period of time to desulfate a battery fully. The battery regenerator is also used for measuring total lifespan of battery (i.e) charging, discharging, and restoration characteristics for 40-60 hours and it also includes the temperature measurements.

The reformer enable to restore or discharge a large range of battery capacities up to some $\mathrm{V} \sim 150 \mathrm{~V}, 4000 \mathrm{Ah} / \mathrm{C}_{10}$. It removes lead-sulfate $\left(\mathrm{PbSO}_{4}\right)$ generated during discharging with the reformer electric characteristics called shark pulse, reduces lead-sulfate again to increase the weight of electrolyte, and decreases internal resistance to recycle the battery [1].

In actual process, sulfate $\left(\mathrm{PbSO}_{4}\right)$ is the formation of a dense, insulating layer of crystalline lead sulfate on the battery ${ }^{\text {es }}$ plates, a natural process that increases with time and cannot be reversed under normal charging conditions.

\subsection{Battery Measurement System}

BMS is specially designed for monitoring the each cell voltages connected in the battery bank. The measurement is rated for not more than $5 \mathrm{~V}$ per cell and it can monitors continuously for the maximum duration of 10 hours [1]. The experimental setup diagram of BMS measurement is shown in Fig. 1.

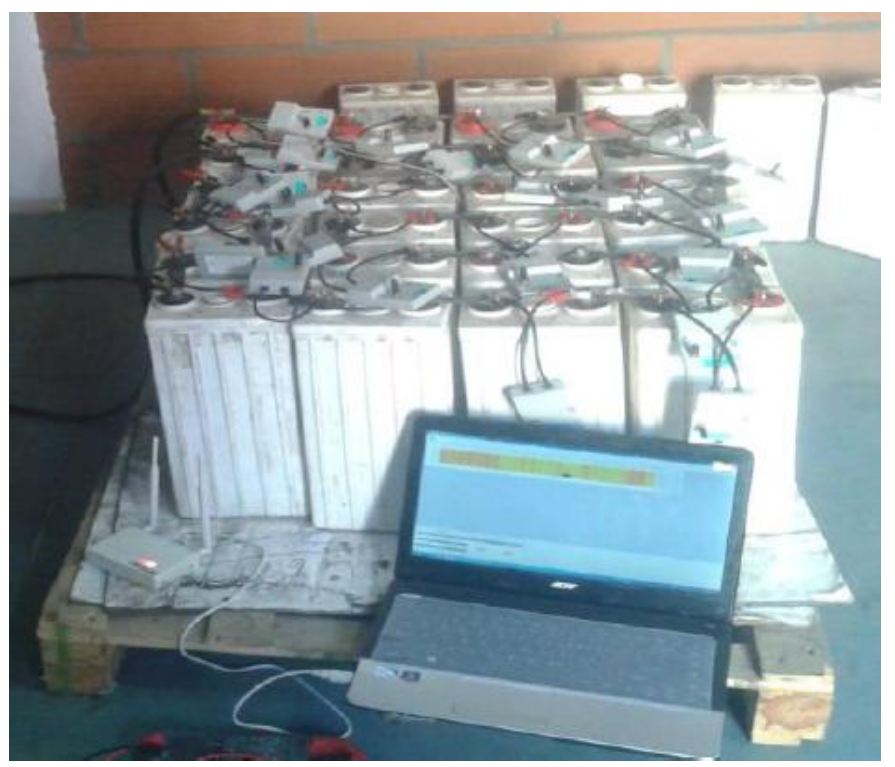

Fig -1: Experimental BMS setup picture.

A BMS provides more accurate information and test result of each cell connected in battery bank. It combines advanced technology to evaluate the battery capacity and to check cell voltage at any time. It enables to minimize their maintenance costs through battery monitoring devices [1]. 


\subsection{Experimental Setup}

The block diagram of experimental setup of battery reformation is shown in Fig. 2. The integration receiver receives the signals from the battery regenerator and BMS. The received signal is fed back to the PC/Laptop for the real time monitoring.

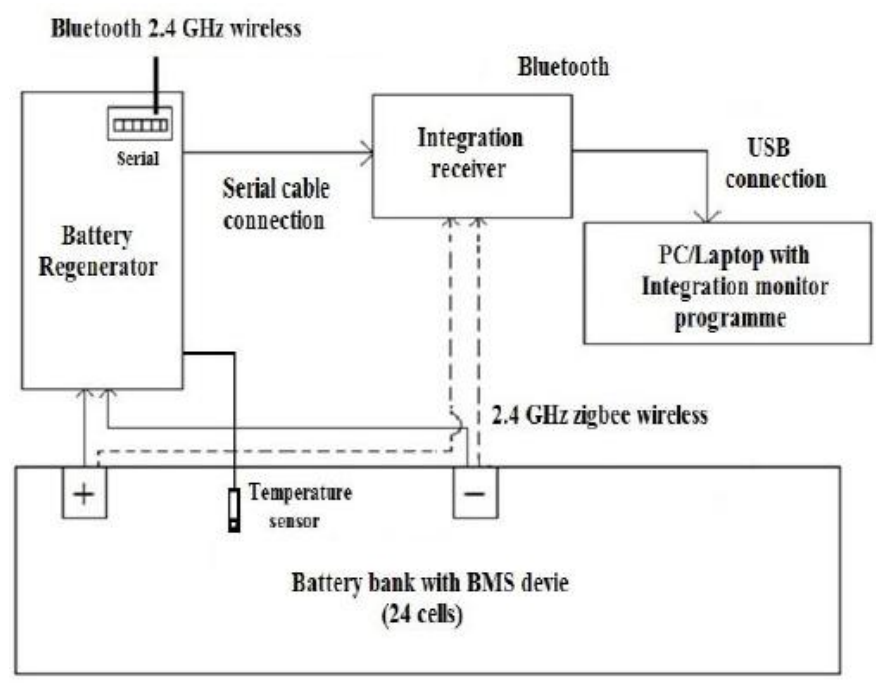

Fig -2: Laboratory setup block diagram.

\subsection{Charging Modes}

The charging and discharging voltage limits are very important for safe operation of battery banks [2]. This is especially important for the VRLA battery for which the preferred charge. The nominal values of current and voltage limits for 300Ah, 48V, 24 cells are given in Table 1.

Table -1: $\mathrm{CC}$ and $\mathrm{CV}$ rating tabulation

\begin{tabular}{|c|c|c|c|c|c|}
\hline \multicolumn{6}{|c|}{ 300Ah Capacity, Cl0 rate, 24 cells, $48 \mathrm{~V}$ battery bank with $80 \%$ DOD } \\
\hline Mode & Voltage (V) & Current $(\mathrm{A})$ & Time (H) & Select & Pulse \\
\hline \multirow{2}{*}{1} & 43.2 & 30 & \multirow{2}{*}{11} & D & \multirow{2}{*}{1} \\
\hline & $1,8 \mathrm{~V} \times 24$ & $300 \mathrm{Ah} \times 10 \%$ & & Discharge & \\
\hline \multirow{2}{*}{2} & 57.6 & 30 & \multirow{2}{*}{8} & $\mathrm{CC}$ & \multirow{2}{*}{1} \\
\hline & $2,4 \mathrm{~V} \times 24$ & $300 \mathrm{Ah} \times 10 \%$ & & Constant Current & \\
\hline \multirow{2}{*}{3} & 57.6 & 15 & \multirow{2}{*}{8} & $\mathrm{CV}$ & \multirow{2}{*}{2} \\
\hline & $2,4 \mathrm{~V} \times 24$ & $300 \mathrm{Ah} \times \mathbf{5 \%}$ & & Constant Voltage & \\
\hline \multirow{2}{*}{4} & 43.2 & 30 & \multirow{2}{*}{11} & D & \multirow{2}{*}{1} \\
\hline & $1,8 \mathrm{~V} \times 24$ & $300 \mathrm{Ah} \times 10 \%$ & & Discharge & \\
\hline \multirow{2}{*}{5} & 57.6 & 30 & \multirow[b]{2}{*}{8} & $\mathrm{CC}$ & \multirow{2}{*}{1} \\
\hline & $2,4 \mathrm{~V} \times 24$ & $300 \mathrm{Ah} \times 10 \%$ & & Constant Current & \\
\hline \multirow{2}{*}{6} & 57.6 & 15 & & CV & \multirow{2}{*}{2} \\
\hline & $2,4 \mathrm{~V} \times 24$ & $300 \mathrm{Ah} \times 5 \%$ & & Constant Voltage & \\
\hline
\end{tabular}

In this tabulation, the number 24 represents the no. of cells connected in the battery bank. Similarly, $1.8 \mathrm{~V}$ represents the lower discharge limit of each cell and $2.4 \mathrm{~V}$ represents the upper charge limit of each cell connected in battery bank.

\section{RESULTS AND DISCUSSION}

The experimental result obtained from the 4 years old age battery bank capacity of $300 \mathrm{Ah}, 48 \mathrm{~V}$ and 24 cells connected in the laboratory experiment system. The discharge voltage profile of 24 cells is shown in Fig. 3. From the experimental result, the cell number \#2 reaches $1.75 \mathrm{~V}$ after 2 hour $30 \mathrm{~min}$ of discharge (Identified as bad cell). Then cell number \#9 and $\# 12$ are no longer in balance with the remaining cells.
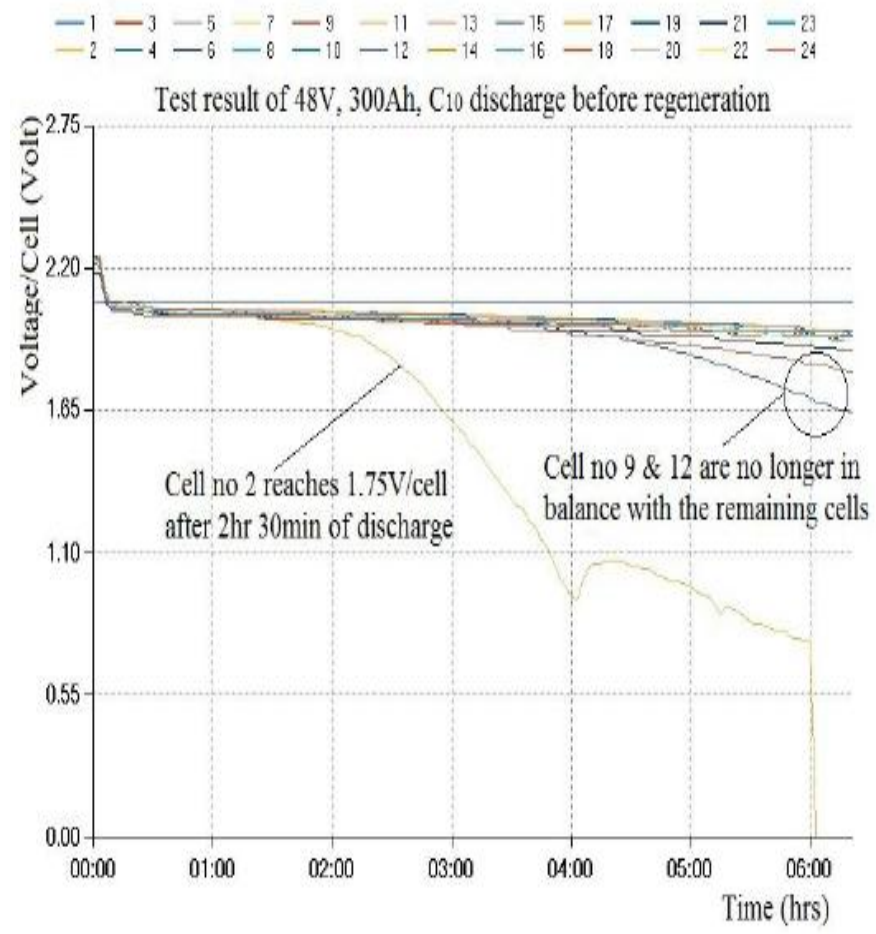

Fig -3: Discharge characteristics of 24 cells, $48 \mathrm{~V}$, and $300 \mathrm{Ah}$ battery bank capacity with $80 \%$ DOD before regeneration (6 hours 30 min duration curve).

This tabulation has obtained from the battery measurement system (BMS). This BMS monitor each cell voltages at every $3 \mathrm{~min}$ or $15 \mathrm{~min}$ time duration through $2.5 \mathrm{GHz}$ zigbee wireless by Integrated Monitor software via Bluetooth. It can monitor maximum time duration of 10 hours for continuous experiment. This real time monitoring software tabulation diagram is shown in Fig. 4. 


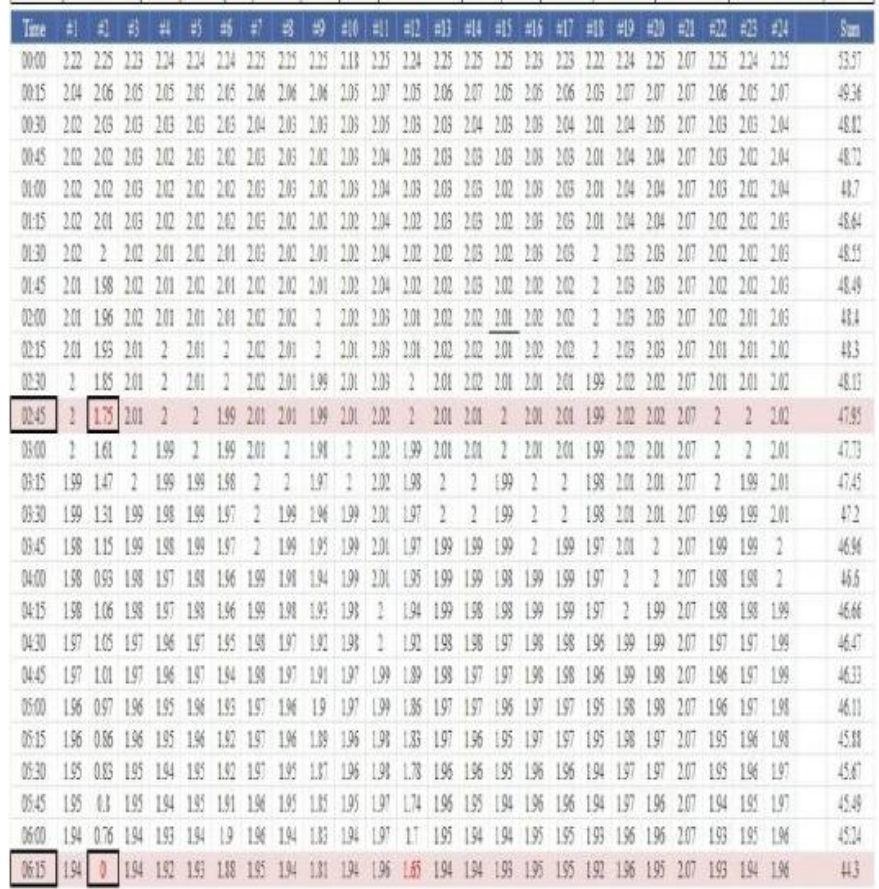

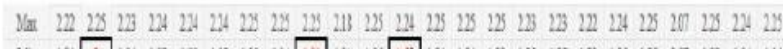

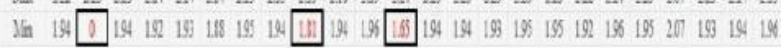

Fig -4: Each cell voltages at 80\% DOD for every 15 min for 6 hours 30 min time duration (Before regeneration).

The main failure of VRLA battery is sulfation occurring in the old aged battery banks. To replace the failure battery it requires huge amount of cost and manpower. Behalf of replacing the old aged/sulfated battery by providing the battery reformer, it reduces the sulfation in the battery and improves the battery capacity performance and lifespan.

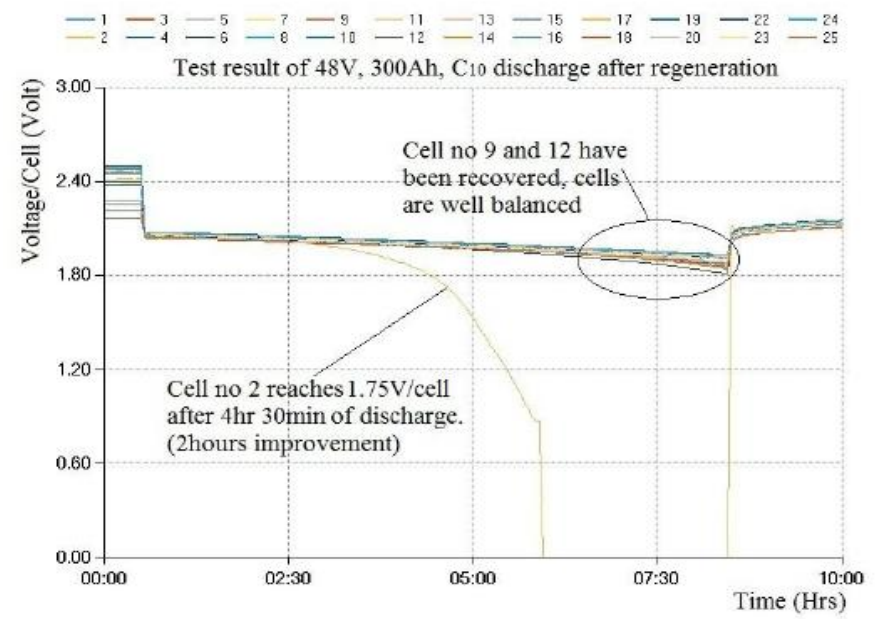

Fig -5: Discharge characteristics of 24 cells, $48 \mathrm{~V}$, and $300 \mathrm{Ah}$ battery bank capacity with $80 \%$ DOD after regeneration (10 hours duration curve).
The experimental result obtained from after regeneration of same system is shown in Fig. 5. From the experimental result of after regeneration, the cell number $\# 2$ reaches $1.75 \mathrm{~V}$ after 4 hour $30 \mathrm{~min}$ of discharge. 2 hours has been improved, but still it is not sufficient. At same time cell number \#9 and \#12 has been recovered and cells are well balanced. This real time monitoring software tabulation diagram for after regeneration is shown in Fig. 6.

\begin{tabular}{|c|c|c|c|c|c|c|c|c|c|c|c|c|c|c|c|c|c|c|c|c|c|c|c|c|}
\hline & ind & 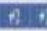 & Di & H & 63. & सा & & wa & 72 & & & & & & 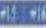 & $6 \mathrm{arl}$ & 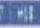 & 191 & we & 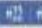 & 530 & & & \\
\hline mii) & (24) & 24120 & 246 & 1) 24 & 242 & 242 & 216 & 14) & 145 & $\pi$ & 142 & $2 \pi$ & 2111 & it 2 & ith 11 & 11. 24 & 120 & 24 & 218 & $250 ?$ & 252 & 24 & 15 & \\
\hline & $245:$ & 202 & 210 & If 21 & 242 & 242 & 20. & 2II & 26 & & 242 & & 20 & (1) & 2612 & 2) 2 & 925 & 24 & 26 & 20. & 202 & 211 & & \\
\hline & $45:$ & 2022 & 226 & $15: 20$ & 242 & $24:$ & 246 & 20 & 245 & & $241 ?$ & 218 & 241 & :1 : & its 21 & 313 & & 24 & & & 202 & 2 & & \\
\hline & $105=$ & 20020 & 2062 & $200 \quad 20$ & 162 & $2 * 2$ & $20:$ & 106 & $20 \%$ & 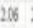 & 20 : & & 2972 & in 2 & 206218 & 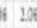 & 6.28 & $23 \pi$ & 20 & 2162 & 2002 & 2 & & \\
\hline ont & 104 & 20020 & $200 ?$ & 3018 & in: & W $?$ & 20 & 106 & $10 x$ & 306 & 30 & 260 & $2 x=1$ & *: & 10615 & $x y$ & 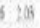 & (207 & 2007 & 2087 & $200 ?$ & 2 & & \\
\hline $0 E$ & 104 & 20520 & 2002 & 20.20 & 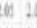 & 1421 & IN & 136 & 1.05 & 268 & $200 \%$ & 216. & $2 x:$ & in & 2065 & 6100 & 62010 & 12) & 295 & 2065 & 2042 & 28 & & \\
\hline & an: & 20020 & 2082 & $2 M 10$ & $165:$ & $i=1$ & $2 x=$ & 108 & 105 & 205 & 2161 & tod & 2032 & 102 & 2031 & of 10 & 130 & 206 & (no & & 2007 & $200 \div$ & & \\
\hline & 162 & 2420 & 2012 & 20220 & 2042 & 292 & 25 & 10 & 166 & & if ? & $206=$ & 251 & 102 & $235=21$ & 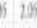 & 620 & DW & & 2012 & 201 & II & & \\
\hline 0.4 & 2012 & 20320 & 2042 & 20: 20 & 2042 & 2912 & 280 & $2 M$ & 206. & 200 & 2002 & 203 & 2032 & $n=2$ & 20.114 & 4.200 & 4210 & $2 x$ & 200 & $2 M=$ & 42 & & & \\
\hline 0?. & 1002 & 20220 & 2012 & $21: 20$ & 2042. & 2.562 & $2 n=$ & 2.4 & 204 & 204. & 202 & $2 M=$ & 242 & wi. & 20424 & 1. 20 & 201 & 200 & 201 & 2032 & 2 & 2 & & \\
\hline & $102=$ & 2420 & 2002 & 20.20 & 161 ? & $2 n+2$ & 245 & 24 & 903 & 20 & 203 & 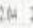 & 294 & $1 * 2$ & $24 \times 14$ & $4: 20$ & 4211 & 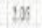 & 204 & as & 18? & ? & & \\
\hline & 201 & 220 & 2012 & 26. 20 & 2031 & in 2 & $2 \mathrm{MI}$ & 101 & 2011 & & & & 2018 & (i) 2 & ain 11 & 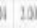 & 21291 & 101 & 2ail & 202 & $202 ?$ & ? & & \\
\hline & 2011 & 19 & & 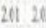 & te: 2 & a) & 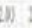 & 203 & 20 & & & & & : & 20) $\leq 1$. & 1) (2) & 4. & $2 x$ & 205 & & & & & \\
\hline 94 & 2011 & 19) 20 & 202 & 20121 & to? & $2 n 2$ & 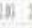 & inf & 2010 & . & 20 & 2. & $2 n 1$ & 11: & 23) 13 & 819 & 21 & $29 \%$ & im & ? & $?$ & 20i & & \\
\hline 0.1. & 21 & Les 20 & 201 & 120 & 20 & 12 & 24. & 20 & 201 & 20 & 206 & 20. & 2022 & (1) 2 & 201.18 & 12.20 & 19 & 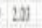 & in & 2012 & 2012 & $20 !$ & & \\
\hline & 21 & 19221 & $201=$ & 120 & 101 & 11 & 101 & 218 & 201 & 100 & 100 & 1 & 221 & 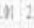 & 21110 & 11) 10 & 10 & 191 & 201 & 201 & 2 & 2 & & \\
\hline & : & 181 20 & 201 & 199: & 21 & 198 & & 201 & ? & 30 & & & 2011 & & 29121 & K 20 & $11 \mathrm{D}$ & 29 & 7.4य & 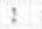 & 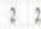 & & & \\
\hline UhI & 1901 & 181 & & 100 & 21 & $14]$ & 311 & 291 & 1 & 201 & 2002 & 201 & 2011 & 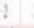 & 211 & 14 I & 18 & 189 & 1 & 11 & to 2 & 201 & & \\
\hline & ] 19 & 17 & 21 & 1818 & 141 & in 1 & 201 & 1 & 199 & 1 & 201 & & 11 & H & 12 & 1 & 193 & 29 & 1. & 1991 & $190 \%$ & 201. & & \\
\hline 0165 & 1981 & 165 & twi 1 & 1618 & (19) 1. & an & 1 & 1 & 19) & 1 & 20 & 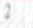 & 1. 1. & 141 & (19) 1 & (1) & 10 & 130 & (9) & 1901 & (9) & & & \\
\hline & 1981 & 19119 & 1991 & 18 & 141 & 191 & 2 & IA & 199 & 199 & 2 & 2 & (9) 1 & 191 & IM) IH & 418 & if 19 & 2 & L & 1501 & 18 & ? & & \\
\hline & (9) 1 . & Lat 18 & 1881 & 21 & In 2 & 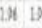 & (D) 1 & 190 & 197 & 1. & 2 & 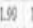 & 1991 & 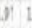 & 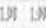 & 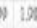 & 105 & 2 & 128 & 1001 & 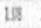 & & & \\
\hline & 197 & 18018 & 1881 & 18015 & 141 & is: 18 & [W & In & in & 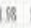 & 1901 & in & 1911 & It 1 & 1010 & n) 19 & 410 & 180 & 197 & 197 & 19: 1 & 18 & & \\
\hline 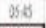 & 196 & 102 & 19. $\mathrm{t}$ & to: 19 & 19) 1 & $D A B$ & |19I & 18 & 106 & 188 & 100 & 10 & IA 1 & th & $19 B$ & $4 \mathrm{~b}$ & 1105 & 19 & 19 & 197 & 19. & IN & & \\
\hline 600 & ] 196 & 0 & 19. 1 & 19. is & if 1 & in 18 & 191 & I I & 195 & 197 & 150 & 9 & 10.1 & me & 1919 & 1317 & 18 & in & 196 & 180 & 161 & 19 & & \\
\hline 65 & 195 & 7 & 181 & tw 15 & IN 1 & 19. $\mathrm{t}$ & [y? & 197 & 191 & 19. & 191 & 6! & 19: 1 & 4. & 19) 17 & 1) 19 & 6196 & 191 & 135 & 180 & 191 & 1 & & \\
\hline & 19? & 014 & 198 & 90 & 180.1 & 19] & 181 & 195. & 194. & 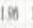 & 151 & t. & IN1 1 & in 1 & 1418 & 719 & 8194 & 197 & (9) & 198 & 1 & 1 & & \\
\hline & 194 & 015 & 18. 1 & 15 & 1911 & 19 & 1 & $19 \%$ & 193 & 18 & & & 1961 & $1 n$ & 195.134 & 3519 & 1.193 & 197 & 194 & 198 & 4 & & & \\
\hline & 194 & 010 & 1951 & th. $\mathrm{ts}$ & tw 1 & Ifif & 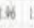 & 191) & 102 & 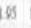 & 1661 & 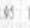 & (9) 1 & $m 1$ & (a) in & 410 & 1108 & 19 & 105 & 104 & 1 & 1 & & \\
\hline & 194) & $A 10$ & 1941 & 1915 & 144 & Ift 18 & 19 & 196 & 191 & 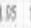 & 1081 & 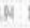 & (b) 1 & W1 1 & the the & 1519 & 410 & 18 & 13 & 1 & 1 & 1 & & \\
\hline & 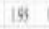 & 1019 & 41 & 9119 & (4) & 1518 & 19: & 194. & 19 & . & & 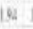 & 1941 & if 1 & 19.10 & 1) 19 & 1) 191 & (19) & (9) & 1 & & 1 & & \\
\hline & & & & $t$ & (4) & 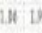 & & |. & 10 & & & & & III 1 & 1) 14 & 41 & 1319 & 191 & ti & & & & & \\
\hline & 191 & 119 & & & & 14.18 & 144 & 198 & 188 & 3 & & & 19) 1 & 1 & 192 18 & 419 & 218 & 194 & 15 & 192 & & 1 & & \\
\hline & L19 & 015 & 15. 1 & 188 & 1912 & L. 1 & Di 1 & LIt & 1.66 & & & & Les 1 & 1) : & 15. 13 & (3) 10. & 2. $1 \mathrm{~s}$ & 6187 & 10 & 191 & 1 & 1 & & \\
\hline & 21 & 21220 & 2N4 28 & 20020 & 104.18 & $18 \times 10$ & 100 & 206 & 207 & $2 h$ & D. & 20. & 264 & 109 & $2162 \mathrm{~W}$ & 4. 12 & 1. 201 & 11.6 & 205 & 205 & 204 & 2U: & & \\
\hline & 10 & 21 & 28 & 242 & 109 & 2022 & 2. & 21 & 41 & 28 & 21 & 13. & $200:$ & 21 & II 20 & $12 x$ & 8 & 21 & 21 & 21) & 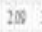 & 11 : & & \\
\hline & 20 & 2084 & 21 21 & 2122 & 2121 & 21121 & 211 & 21II & 2.11 & 21 & LII : & 200 & 112 & III & 21126 & 621 & 1 & 2111 & 2.1I & 211 & 21 & 2 & & \\
\hline & 212 & 11 21 & 21121 & 21321 & 111 & 211 & & 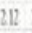 & 212 : & 211 & U18 & 2 & 2112 & 21 & 21211 & 1211 & II & 112 & ? & 1 & $2 \mathrm{II}$ & l & & \\
\hline & & 211 & & 2.11 4 & 11? 1 & $11 ?$ & & & $\theta$ & & 1119 & & & & 213.211 & 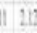 & & & & & & 2 & & \\
\hline & 2 & 212 & & 21511 & 111 & 111 21 & -1 & 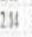 & & & 2iI & & & & 214 & & & 14 & & & & 2 & ? & \\
\hline & 2122 & 212. 2 & 21321 & 261 & L. 1 & Ift & & & 215 & & It ? & & 211 & & & & 211 & 1215 & & 215 & 2.4 & 211. & & \\
\hline & & & & & & & & & & & & & & & & & & & & & & & & \\
\hline & & & & & & & & & & & & & & & & & & & & & & & & \\
\hline
\end{tabular}

Fig -6: Each cell voltages at $80 \%$ DOD for every $15 \mathrm{~min}$ for 10 hours time duration (After regeneration).

The continuous two cycle es characteristics of discharge, constant-current charge, constant-voltage charge of 300Ah, $48 \mathrm{~V}$ battery bank for 39 hours duration curves is shown in Fig. 7. This graph is drawn with respect to voltage (red), current (orange), and temperature (blue) vs. time for real time experiment. Explanation of graph starts from left to right direction. First one is discharge, charge and equalization for before regeneration. After regeneration is (starts from no. 4) again discharge, charge and equalization. From this graph, comparison of the discharge curves of number \#1 and \#4 shown in Fig. 7.

Discharge no. 1 - time duration approx 7 hours at -195.6Ah. Discharge no. 4 - time duration approx 8 hours at $-223.4 \mathrm{Ah}$ 
for without replacing the bad cell \#2. Extra 1 hour backup is obtained by without replacing cell number \#2.

In VRLA 300Ah capacity battery bank, the cell number \#2 is the weak link in this battery string arrangement. After regeneration all cells are perfectly balanced (not including cell number \#2), then the discharge process have added 1 hour and 30 minutes more of backup regardless of the bad cell which identified in cell number \#2. If we would remove and replace cell number \#2, it will reach at least 1 hour 30 min more of backup. This results shown that the battery bank will reach the maximum capacity when applying a $\mathrm{C}_{10}$ discharge test.

UPS backup: 3 hours and 30 minutes before regeneration. UPS backup: 5 hours after regeneration.

If replacing the cell number \#2 backup would be around more than 7 hours is possible. Similarly, same procedure is conducted for various capacity ratings of 550Ah, 577Ah, 660Ah, 682Ah with $48 \mathrm{~V}$ battery banks. The performance comparison tabulation is given in Table 3 .
Table 2: Case study Analysis

\begin{tabular}{|c|c|c|c|}
\hline \multicolumn{3}{|c|}{ Case - 1 - 300Ah } \\
\hline S.No & $\begin{array}{c}\text { Total test } \\
\text { hours }\end{array}$ & $\begin{array}{c}\text { Improved } \\
\text { capacity (Ah) }\end{array}$ & $\begin{array}{c}\text { Per unit } \\
\text { consumption } \\
\text { (kWhr) }\end{array}$ \\
\hline 1 & $39: 02: 47$ & 27.80 & 0.0820 \\
\hline
\end{tabular}

\begin{tabular}{|c|c|c|c|}
\hline \multicolumn{3}{|c|}{ Case - 2 - 550Ah } \\
\hline S.No & $\begin{array}{c}\text { Total test } \\
\text { hours }\end{array}$ & $\begin{array}{c}\text { Improved } \\
\text { capacity (Ah) }\end{array}$ & $\begin{array}{c}\text { Per unit } \\
\text { consumption } \\
\text { (kWhr) }\end{array}$ \\
\hline 1 & $46: 31: 14$ & 68.80 & 0.7433 \\
\hline 2 & $32: 44: 48$ & 137.30 & 0.9083 \\
\hline
\end{tabular}

\begin{tabular}{|c|c|c|c|}
\hline \multicolumn{3}{|c|}{ Case - 3 -682Ah } \\
\hline S.No & $\begin{array}{c}\text { Total test } \\
\text { hours }\end{array}$ & $\begin{array}{c}\text { Improved } \\
\text { capacity (Ah) }\end{array}$ & $\begin{array}{c}\text { Per unit } \\
\text { consumption } \\
\text { (kWhr) }\end{array}$ \\
\hline 1 & $42: 24: 08$ & 64.60 & 0.3478 \\
\hline 2 & $38: 54: 17$ & 110.90 & 0.5813 \\
\hline
\end{tabular}

From the above experiment data of obtained results shows that, per unit consumption for performance improvement purely depends on the ampere-hour improvement for before and after regeneration of the field level battery banks of various capacities. This result is shown in Table 2 and the performance analysis shown in Chart 1. Total unit power consumption is not depends on the battery bank capacity ratings. It ${ }^{\text {ees }}$ purely depends on electrical charging for fully desulfate the particular battery bank (i.e) ampere-hour build up in the battery bank capacity.

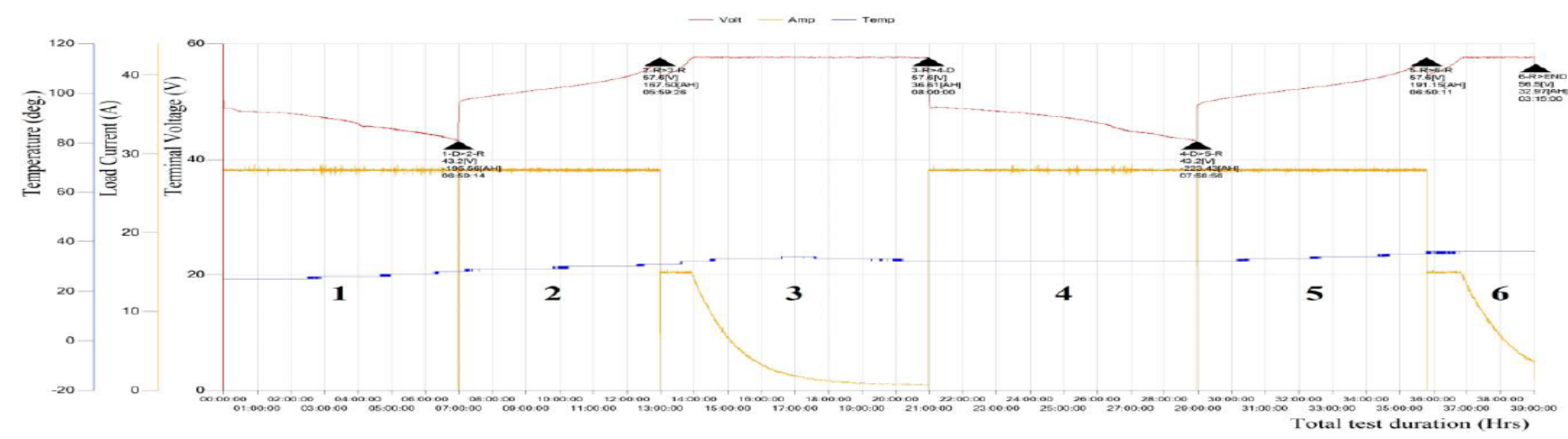

\begin{tabular}{|c|c|c|c|c|c|c|c|c|c|c|}
\hline Index & Method & Pulse & Setup Time & $\begin{array}{c}\text { Elapsed } \\
\text { Time }\end{array}$ & $\begin{array}{l}\text { Setup } \\
\text { Volt }\end{array}$ & $\begin{array}{l}\text { Max } \\
\text { Volt }\end{array}$ & Min Volt & $\begin{array}{l}\text { Setup } \\
\text { Amp }\end{array}$ & Ah & Temperature \\
\hline 1 & Discharge & 1 & 11:00:00 & $06: 59: 14$ & 43.2 & 53.0 & 43.2 & 28.0 & -195.6 & +28 \\
\hline 2 & Restoration & 1 & 08:00:00 & $05: 59: 26$ & 57.6 & 57.6 & 46.1 & 28.0 & 167.5 & +31 \\
\hline 3 & Restoration & 2 & 08:00:00 & 08:00:00 & 57.6 & 57.7 & 53.9 & 15.0 & 36.6 & +32 \\
\hline 4 & Discharge & 1 & 11:00:00 & $07: 58: 56$ & 43.2 & 57.3 & 43.2 & 28.0 & -223.4 & +32 \\
\hline 5 & Restoration & 1 & 08:00:00 & $06: 50: 11$ & 57.6 & 57.6 & 44.7 & 28.0 & 191.2 & +35 \\
\hline 6 & Restoration & 2 & 08:00:00 & $03: 15: 00$ & 57.6 & 57.7 & 54.1 & 15.0 & 33.0 & +36 \\
\hline \multicolumn{11}{|c|}{$39: 02: 47$} \\
\hline
\end{tabular}

Fig -7: Charging, discharging and lifespan characteristics curve of 48V, 300Ah battery bank capacity with 80\% DOD - Before and after regeneration for 39 hours duration curves. 
Table -3: Performance Analysis Comparison

\begin{tabular}{|c|c|c|c|c|c|c|c|c|c|c|}
\hline \multirow[b]{2}{*}{$\begin{array}{l}\text { S. } \\
\text { No }\end{array}$} & \multirow{2}{*}{$\begin{array}{c}\text { BB } \\
\text { capacity } \\
\text { (Ah) }\end{array}$} & \multirow{2}{*}{$\begin{array}{c}\text { Total } \\
\text { experiment } \\
\text { Time (Hrs) }\end{array}$} & \multirow{2}{*}{$\begin{array}{l}\text { Operating } \\
\text { temperature } \\
\text { ranges }\left({ }^{\circ} \mathrm{C}\right)\end{array}$} & \multicolumn{2}{|c|}{ Before reformation } & \multicolumn{2}{|c|}{ After reformation } & \multicolumn{2}{|c|}{$\begin{array}{l}\text { Performance } \\
\text { Improvement }\end{array}$} & \multirow{2}{*}{$\begin{array}{c}\text { Total power } \\
\text { consumption } \\
\text { (kWhr) }\end{array}$} \\
\hline & & & & $\begin{array}{c}\text { Capacity } \\
\text { (Ah) }\end{array}$ & $\begin{array}{c}\text { Backup } \\
\text { time } \\
\text { (Hrs) }\end{array}$ & $\begin{array}{c}\text { Capacity } \\
\text { (Ah) }\end{array}$ & $\begin{array}{c}\text { Backup } \\
\text { time } \\
\text { (Hrs) }\end{array}$ & $\begin{array}{c}\text { Capacity } \\
\text { (Ah) }\end{array}$ & $\begin{array}{c}\text { Backup } \\
\text { time } \\
\text { (Hrs) }\end{array}$ & \\
\hline 1 & 300 & $39: 02: 47$ & +28 to +36 & -195.60 & $06: 59: 14$ & -223.40 & $07: 58: 56$ & -27.80 & $0: 59: 42$ & 3.2898 \\
\hline \multirow{3}{*}{2} & 550 & $32: 44: 48$ & +27 to +51 & -171.70 & 03:07:29 & -309.00 & $05: 37: 19$ & -137.30 & $2: 29: 50$ & 29.9742 \\
\hline & 577 & $37: 20: 47$ & +22 to +51 & -308.20 & $05: 20: 47$ & -461.30 & 08:00:00 & -153.10 & $2: 39: 13$ & 28.0182 \\
\hline & 550 & $46: 31: 14$ & +28 to +56 & -254.60 & 04:38:01 & -323.40 & $05: 53: 13$ & -68.80 & $1: 15: 12$ & 34.1961 \\
\hline \multirow{3}{*}{3} & 660 & $34: 29: 11$ & +36 to +51 & -280.70 & $04: 16: 26$ & -410.80 & $06: 13: 45$ & -130.10 & $1: 57: 19$ & 23.4845 \\
\hline & 682 & $38: 54: 17$ & +33 to +52 & -464.00 & $06: 48: 25$ & -574.90 & $08: 25: 59$ & -110.90 & $1: 37: 34$ & 22.6711 \\
\hline & 682 & $42: 24: 08$ & +36 to +50 & -594.70 & $08: 43: 37$ & -659.30 & $09: 40: 31$ & -64.60 & $0: 56: 54$ & 14.6109 \\
\hline
\end{tabular}

In this tabulation, the negative sign (-) indicates that ampere-hours at the time of discharge, $\mathrm{BB}=\mathrm{Battery}$ bank, ${ }^{\circ} \mathrm{C}=$ Operating temperature in degrees, $\mathrm{Ah}=$ Battery bank capacity rating, $\mathrm{V}=$ Volt, $\mathrm{A}=$ Ampere, $\mathrm{H}=$ Hours, $\mathrm{kWhr}=$ Unit consumption in kilowatt hours.

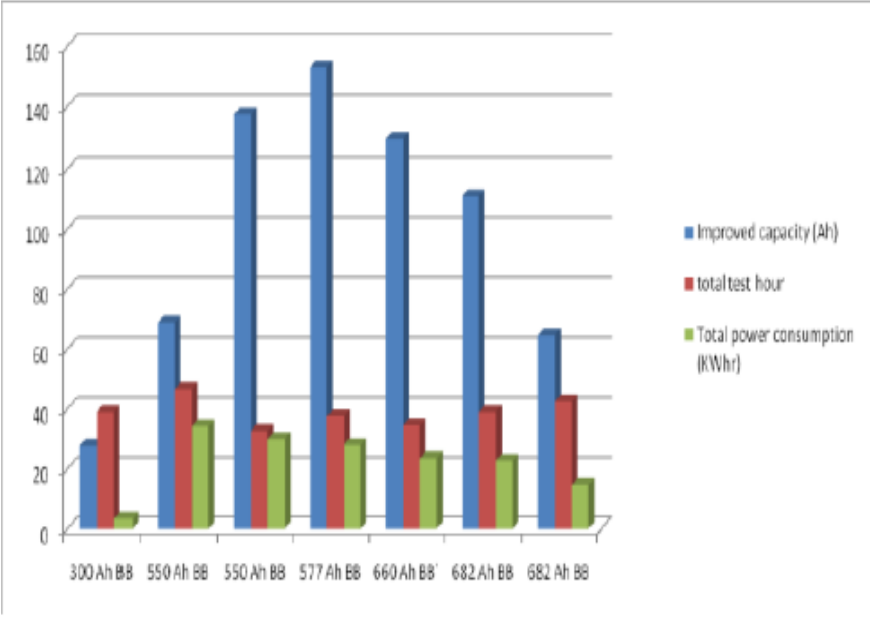

Chart -1: Performance analysis - Various capacity rating battery banks.

\section{CONCLUSIONS}

By analyzing charging, discharging, lifespan and thermal characteristics of various capacity rating battery banks, suitable battery bank capacity rating for particular battery reformation applications can be chosen. Enormous amount of power can be saved by after reformation of existing old age battery banks and replacing the identified bad/death cell in the battery string by energy efficient batteries obtained. From the experimental results it is observed that the power consumption, heat emission and fuel consumption of batteries is less when compared to before reformation of various capacitates battery banks. The improved ampere-hour rating and backup time level of reformatted battery banks hold best performance when compared to previous one and improved power saving for future.

\section{REFERENCES}

[1]. Battery Regenerating Technique and BMS Operating Manual, MarooMCS Inc., Korea, 2010. [2]. L. David, and B.R. Thomas, "Handbook of Batteries," McGraw-Hill Companies, Inc., New York, 2002. [3]. G. Jian, B. Sen, C. Jian, W. Xianzhang, and X. Haifeng, "An Innovative VRLA Battery Solution for Energy Saving \& Emission Reduction," Proc. 34th IEEE International Telecommunications Energy Conference (INTELEC „12), pp.1-5, 2012.

[4]. W. Jian, W. Zhengbin, D. Xianquan, Q. Songhua, and Y. Xiaoping, "Temperature Characteristics Improvement of Power Battery Module for Electric Vehicles," Proc. IEEE International Conference on Vehicle Power and Propulsion Conference (VPPC „13), pp.1-4, 2013. [5]. A. Marongiu, A. Damiano, and M. Heuer "Experimental Analysis of Lithium Iron Phosphate Battery Performances," Proc. IEEE International Symposium on Industrial Electronics (ISIE „10), pp.3420-3424, 2010. [6]. C. Sen, and N.C. Kar, "Analysis of the Battery Performance in Hybrid Electric Vehicle for Different Traction Motors," Proc. IEEE Conference on Electrical Power and Energy Conference (EPEC „09), pp.1-6, 2009. [7]. A. Mariani, K. Thanapalan, P. Stevenson, and J. Williams, "Techniques for Estimating the VRLA Batteries Ageing, Degradation and Failure Modes," Proc. 19th International Conference on Automation and Computing (ICAC „13), pp.1-5, 2013.

[8]. D. Meekhun, V. Boitier, and J.M. Dilhac, "Charge and Discharge Performance of Secondary Batteries According to 
Extreme Environment Temperatures," Proc. 35th IEEE Annual Conference on Industrial Electronics Conference (IECON '09), pp.266-270, 2009.

[9]. T. Guena, and P. Leblanc, "How Depth of Discharge Affects the Cycle Life of Lithium-Metal-Polymer Batteries," Proc. 28th Annual International Telecommunications Energy Conference (INTELEC '06), pp.1-8, 2006. [10]. C. Cedric, S. Adnan, A.D. Ahmed, and S.M. Muyeen, "Modeling and Analysis of Battery Performance for Renewable Energy Application," Proc. 15th European Conference on Power Electronics and Applications (EPE „13), pp.1-10, 2013.

\section{BIOGRAPHIES}

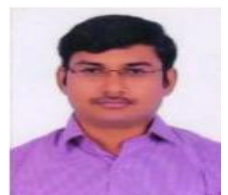

J. Vishnupriyan received his B.E degrees in Electrical and Electronics Engineering from KLN College of Engineering, Madurai, Tamilnadu, India in the year 2010 and currently doing his M.Tech degree in Control and Instrumentation Engineering at Thiagarajar College of Engineering, Madurai, Tamilnadu, India. His research interest includes battery measurement system and energy efficient analysis.

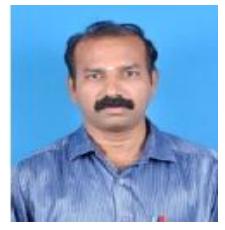

P.S. Manoharan received his B.E and M.E degrees in Electrical Engineering from Thiagarajar College of Engineering, Madurai, Tamilnadu, India in the year 1999 and 2001 respectively, and $\mathrm{Ph} . \mathrm{D}$ degree in Power System from Anna University Chennai, Tamilnadu, India in the year 2009. He is currently working as an Associate Professor with the Department of Electrical and Electronics Engineering, Thiagarajar College of Engineering, Madurai, Tamilnadu, India. He has published more than 50 papers in International Journals/Conferences. His research interest includes power system, evolutionary algorithm and solar energy.

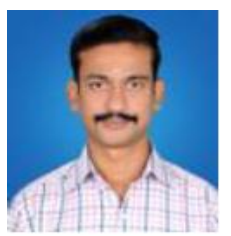

V. Seetharaman received his B.E degree in Electrical and Electronics Engineering from Sethu Institute of Technology, Madurai, Tamilnadu, India in the year 2006 and M.E degree in Power Electronics and Drives from PSNA College of Engineering and Technology, Dindigul, Tamilnadu, India in the year 2008. He is working as an Assistant Professor with the Department of Electrical and Electronics Engineering, Thiagarajar College of Engineering, Madurai, Tamilnadu, India. Now he is currently doing his research in the area of analysis of batteries by soft switching techniques. 